\title{
Herramienta de Evaluación de Tecnologías para la adquisición de equipos biomédicos
}

\author{
Chavarría T. ${ }^{*}$, Molina T. \\ Grupo de Investigación en Tecnologías en Salud Universidad CES, Medellín, Colombia
}

\begin{abstract}
Resumen - La Evaluación de Tecnologías en Salud es una práctica que permite a las instituciones de salud valorar de forma integral las consecuencias del uso de una tecnología, sin embargo, la falta de estandarización y de metodologías que guíen el proceso sigue siendo un reto por superar. Este trabajo presenta el desarrollo y la validación de una herramienta de evaluación de tecnologías en salud la cual se realizó en tres fases: primero se desarrolló la herramienta que permite valorar el proceso de adquisición de equipos biomédicos, después se conformó un grupo de discusión para obtener diferentes percepciones frente a la metodología empleada, el diseño y desarrollo de la herramienta para finalmente implementarla mediante casos de estudio evaluando su uso real y aplicando una encuesta que permitió valorar su utilidad en los procesos de adquisición de equipos biomédicos. Se ponderó la importancia de cada tipo de evaluación de tecnologías seleccionada y se desarrollaron los módulos para las evaluaciones técnica, clínica, económica y ética, social y organizacional. Por su parte, la validación con expertos demostró la utilidad de la herramienta en los procesos de selección y adquisición de equipos biomédicos de manera que se disminuyan costos y se realicen mejores inversiones.
\end{abstract}

Palabras clave - Adquisición de equipos biomédicos, Evaluación de Tecnologías en Salud, tecnologías en salud.

\section{Health Technology Assessment Tools for Biomedical Equipment ACQUISITION}

\begin{abstract}
Health Technologies Assessment is a practice that enablesthe evaluation of the consequences of the use of a technology. However, the lack of standardization and methodologies that guide the process remains a challenge. This paper presents the development and validation of a Health Technology Assessment tool which process was carried out in three phases. First, the tool was developed to assess the acquisition process of medical equipment, after which a discussion group was formed to obtain different perceptions regarding the tool. Finally,was the implementation through case studies, evaluating its real use and applying an evaluation survey to measure its usefulness in the processes of acquisition of medical equipment. The importance of each type of technology assessment was weighed and modules for technical, clinical, economic, ethical, social and organizational evaluation were developed. In addition, validation with experts demonstrated the usefulness of the tool in the selection and acquisition processes of medical equipment in a way that will reduce costs and enable better investments.
\end{abstract}

Keywords - Medical equipment acquisition, Health Technologies Assessment, Health Technologies.

\% Dirección para correspondencia: tchavarria@ces.edu.co

DOI: https://doi.org/10.24050/19099762.n21.2017.1167 


\section{Ferramenta de Avaliação de Tecnologias Para a AQuisição de EQUIPAMENTOS BIOMÉDICOS}

Resumo - A Avaliação de Tecnologias em Saúde é uma prática que permite às Instituições de Saúde valorizar de forma integral as consequências do uso de uma tecnologia, no entanto, a falta de estandardização e de metodologias que guiem o processo segue sendo um desafio por superar. Este trabalho apresenta o desenvolvimento e a validação de uma ferramenta de Avaliação de Tecnologias em Saúde a qual se realizou em três fases: primeiro desenvolveu-se a ferramenta que permite valorizar o processo de aquisição de equipamentos biomédicos, depois se conformou um grupo de discussão para obter diferentes percepções em frente à metodologia empregada, o desenho e desenvolvimento da ferramenta para finalmente implementá-la mediante casos de estudo avaliando seu uso real e aplicando uma pesquisa que permitiu valorizar sua utilidade nos processos de aquisição de equipamentos biomédicos. Ponderou-se a importância de cada tipo de avaliação de tecnologias selecionadas e desenvolveram-se os módulos para as avaliações técnica, clínica, económica, ética, social e organizacional. Por sua vez, a validação com experientes demonstrou a utilidade da ferramenta nos processos de seleção e aquisição de equipamentos biomédicos de maneira que se diminuam custos e se realizem melhores investimentos.

Palavras-chaves-Aquisição de equipamentos biomédicos, Avaliação de Tecnologias em Saúde, Tecnologias em Saúde.

\section{INTRODUCCIÓN}

$\mathrm{L}$ as tecnologías en salud (TS) son todos los equipos y dispositivos biomédicos y quirúrgicos usados en la atención médica, esto incluye medicamentos, sistemas de soporte de la organización como los de información y al mismo tiempo procedimientos médico - quirúrgicos [1].

Los equipos biomédicos se han convertido en una poderosa herramienta para resolver diversos problemas de la salud humana, mejorando las posibilidades de diagnosticar y tratar más enfermedades, sin embargo la incorporación de estas tecnologías implica una gran responsabilidad por parte de las instituciones de salud (IS) debido a los diferentes riesgos que impactan a los pacientes y usuarios, situación que eleva los índices de eventos adversos.

El proceso de Evaluación de Tecnologías en Salud (ETES) es una práctica que permite a las IS valorar de forma integral las consecuencias técnicas, clínicas, sociales, económicas y éticas derivadas del uso de una tecnología [2].

La adquisición de equipos biomédicos es una etapa del proceso de ETES donde se encuentran los planes y programas de compras de los equipos según necesidades, prioridades y disponibilidad de recursos [3], la adecuada incorporación de la tecnología biomédica para la prestación de los servicios de salud, garantiza la calidad en la prestación y la seguridad de los pacientes por medio del logro de relaciones positivas de costo - beneficio y costo - efectividad y con ello la consecución de convenientes resultados en salud [4].

Sin embargo, a pesar de la importancia de este proceso, la mayoría de las decisiones de adquisición de tecnologías son tomadas por áreas administrativas sin participación multidisciplinar con el área médica y el área técnica o de ingeniería, esto se refleja en inversiones que producen adquisiciones que al poco tiempo no responden a las verdaderas necesidades de la comunidad a atender $y$ en el peor de los casos, generan incompatibilidad con la infraestructura hospitalaria, altos gastos de mantenimiento, accidentes tecnológicos por mal uso debido a la falta de entrenamiento y poco impacto funcional frente a los usuarios [5].

Esta investigación buscó desarrollar y validar la utilidad de una herramienta de evaluación de tecnologías (HETES) para la adquisición de equipos biomédicos en IS, se desarrolló la herramienta y se sometió a validación por parte de un grupo de discusión y de expertos mediante el uso de casos de estudio.

\section{MetodologíA}

El estudio se realizó en tres etapas: la primera consistió en el desarrollo de una HETES que permite valorar el proceso de adquisición de equipos biomédicos en las IS, en la segunda se conformó un grupo de discusión para obtener diferentes percepciones frente a la metodología de ETES empleada, el diseño y desarrollo de la herramienta, en la tercera se implementó la herramienta mediante casos de estudio para evaluar el uso real de la herramienta y se aplicó una encuesta para valorar la utilidad de la HETES desarrollada en los procesos de adquisición de equipos biomédicos.

a) Desarrollo de una herramienta tipo software para la ETES en el proceso de adquisición de equipos biomédicos

En esta parte del proyecto se desarrolló un prototipo de HETES basada en las metodologías propuestas por la OMS [6], CENETEC [7] y una encuesta aplicada a un grupo de expertos en ETES.

Para el desarrollo del software, se establecieron los requisitos funcionales de la herramienta, se diseñó el árbol de objetivos, el establecimiento de funciones y el diseño en detalle. 
Para esta etapa igualmente se aplicó una encuesta en línea con el objetivo de valorar la pertinencia e importancia de las variables y de los tipos de evaluación seleccionados de las metodologías ETES de la OMS y CENETEC, los resultados de la encuesta fueron analizados estadísticamente para asignarle a cada uno de los tipos de evaluaciones seleccionadas un valor porcentual que indique la importancia de este tipo de evaluación en el proceso de adquisición, de la misma manera, a cada parámetro dentro de los tipos de evaluación se le asignó un valor porcentual que visualice su grado de importancia en el proceso de ETES.

La HETES arroja finalmente un valor cuantitativo para cada tipo de tecnología biomédica que la IS esté considerando adquirir. Este valor es el que permite tomar decisiones de compra basadas en la realización de un proceso estandarizado.

b) Validación de la herramienta diseñada y percepción de futuros usuarios

Se conformó un grupo de discusión a través de una evaluación de expertos.

Mediante un enfoque cualitativo y un modelo de investigación colaborativa se propuso la valoración inicial de expertos de la HETES propuesta y se identificaron las necesidades, fortalezas y oportunidades de mejora para el prototipo.

c) Validación de la funcionalidad y utilidad de la HETES

Se aplicó un caso de estudio a cinco (5) expertos en ingeniería clínica de IS seleccionadas de Medellín, esto permitió evaluar la funcionalidad y definir otros lineamientos y requisitos a considerar en el diseño. Para la selección de los expertos se tuvo en cuenta el grado de interés y compromiso de estos, así como el grado de experiencia en la realización de procesos de evaluación y adquisición de tecnología biomédica.

Igualmente, se aplicó una encuesta con el fin de evaluar la utilidad de la herramienta en las áreas de ingeniería clínica de las instituciones de salud de la ciudad de Medellín.

\section{Resultados}

A. Desarrollo de una herramienta tipo software para la ETES en el proceso de adquisición de equipos biomédicos:

Para la definición de las actividades más relevantes en el procedimiento de ETES en la etapa de adquisición fue necesario un recorrido por la bibliografía, resaltando los tipos de evaluación que se realizan y las subcategorías de estos tipos, analizando al mismo tiempo cómo cambian las actividades y la ETES con el tiempo.

Se tomaron los cuatro (4) grandes tipos de ETES sugeridas por la bibliografía: evaluación clínica, evaluación técnica, evaluación económica y evaluación ética, social y organizacional y a su vez, se identificaron los criterios a evaluar en cada uno.

Para definir los tipos de evaluación y subcategorías del procedimiento de ETES se diseñó y aplicó una encuesta en línea empleando la herramienta MonkeySurvey, el instrumento fue diligenciado por veintidós (22) expertos en ETES de Colombia y México.

La encuesta permitió calificar la importancia de cada uno de los tipos de ETES de manera cuantitativa de 1 a 4, siendo 1 la opción de "No es importante", 2 la opción de "Poco importante", 3 la opción de "Importante" y 4 la opción de "Muy importante".

De esta forma, se obtuvieron los resultados mostrados en la Tabla 1 .

Tabla 1. Calificación promedio de cada tipo de evaluación

\begin{tabular}{|cc|}
\hline TIPO DE EVALUACIÓN & $\begin{array}{c}\text { CALIFICACIÓN } \\
\text { PROMEDIO }\end{array}$ \\
\hline Evaluación clínica & 3,68 \\
Evaluación técnica & 3,82 \\
Evaluación económica & 3,59 \\
Evaluación ética, social y & \\
organizacional & 2,95 \\
\hline
\end{tabular}

Al convertir cada calificación en porcentajes, se obtiene un peso porcentual para cada uno de los tipos de evaluaciones seleccionados, como lo muestra la Tabla 2.

Tabla 2. Porcentaje de importancia de cada tipo de evaluación

\begin{tabular}{|cc|}
\hline TIPO DE & PORCENTAJE DE \\
EVALUACIÓN & IMPORTANCIA \\
\hline Evaluación clínica & $26 \%$ \\
Evaluación técnica & $27 \%$ \\
Evaluación económica & $26 \%$ \\
Evaluación ética, social y & \\
organizacional & $21 \%$ \\
\hline
\end{tabular}

Se compararon los datos presentados en la Tabla 2 con los obtenidos con los procedimientos de adquisición de otras organizaciones y se encuentran diferencias en los porcentajes pero se conserva el orden jerárquico de importancia, siendo la evaluación técnica la primera, seguida por la clínica, la económica y por último la evaluación ética, social y organizacional [8]. 
La evaluación técnica es un aspecto es muy importante y debe tener el mayor peso al momento de tomar una decisión de adquisición de tecnología ya que evalúa condiciones relacionadas con el ámbito funcional y técnico del equipo [9].

Para el desarrollo del software para la ETES en el proceso de adquisición de equipos biomédicos, se empleó el framework WaveMaker, programación en JAVA y una base de datos en MySQL, se programaron los módulos para cada una de las etapas del proceso para los tipos de evaluación de tecnologías seleccionados, el funcionamiento de la HETES comienza cuando el usuario ingresa las diferentes tecnologías (u opciones de equipos) a evaluar, iniciando por la evaluación técnica, posteriormente, el sistema permite valorar y comparar cada tecnología desde las evaluaciones económica, clínica, ética, social y organizacional; finalmente el software se encarga de ponderar cada una de las respuestas para dar una recomendación.

\section{Evaluación técnica}

La evaluación técnica se inicia cuando el usuario ingresa las especificaciones técnicas que considera importantes para la tecnología que está evaluando, posteriormente debe valorarlas con una calificación de 1 a 5 (donde 5 es una calificación MUY ALTA para el equipo en dicha especificación, 4 es calificación ALTA, 3 es calificación MODERADA, 2 es calificación MALA y 1 es DEFICIENTE) en la casilla denominada "nota" del formulario que se presenta en la Fig. 1.

El sistema finalmente calculará y presentará el valor de la evaluación técnica para cada uno de los equipos.

\section{Evaluación económica}

La evaluación económica contempla no solo el valor de la compra del equipo médico sino también su depreciación, valorización, mantenimiento y demás movimientos contables [10].

La necesidad de realizar una evaluación económica surge de la condición de administrar los recursos que en la mayoría de las instituciones de salud de nuestro país son limitados; es muy importante mencionar que los resultados de esta evaluación se deben contrastar con la evaluación de la tecnología en términos de seguridad, eficiencia y efectividad otorgados por la evaluación técnica [11].

La evaluación económica, busca ofrecer pautas que permitan elegir objetiva y sistemáticamente la mejor opción de un grupo de soluciones a cierta necesidad (oportunidad) desde el punto de vista monetario [11].

Para el análisis económico de la adquisición de la nueva tecnología, se propone una evaluación de la inversión que permita tener en cuenta la rentabilidad, el nivel de riesgo y la liquidez.

Una inversión está caracterizada por tres parámetros: inversión inicial (K), vida del equipo (n) y flujo de caja (R) [12].

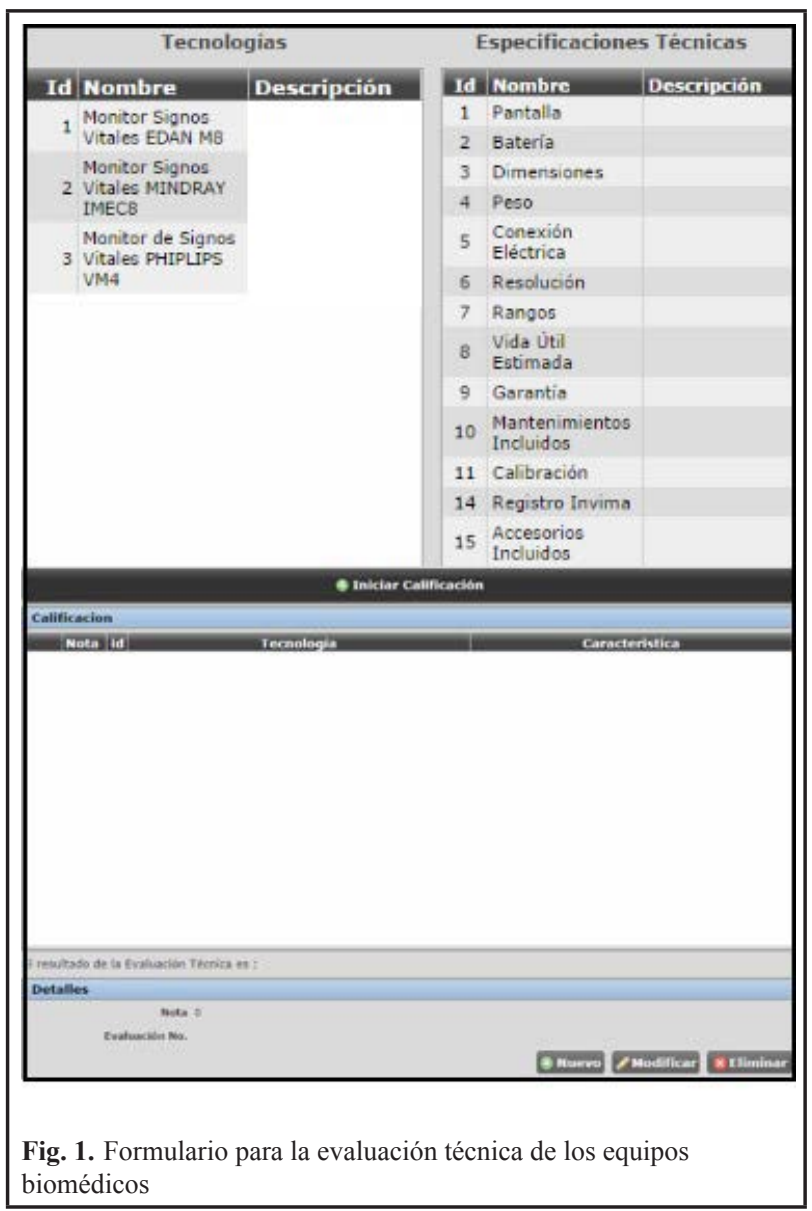

Usando (1) se calcula el flujo de caja como resultado de los ingresos derivados del uso del equipo menos los pagos.

$$
\text { Flujo de caja }(R)=\text { Ingresos }(I)-\text { Pagos }(P)
$$

El flujo de caja para los años de vida del equipo en años sucesivos se calculó usando (2).

$$
\text { Valor futuro }=\quad \begin{gathered}
n \\
\text { Valor actual } x(1+i)
\end{gathered}
$$

$i$ es la inflación, $n$ es el número de años para los cuales se hace el análisis.

Mediante (3) calculamos la rentabilidad de la nueva tecnología usando el VAN (Valor Actual Neto), para lo 
cual restamos la inversión del equipo $(\mathrm{K})$ del flujo de caja año tras año [12].

$$
\begin{gathered}
F C 1 F C 2 V R+F C n \\
V A N=- \text { Inversión }+--\mp---\cdots--- \\
12 n \\
(1+i)(1+i)(1+i)
\end{gathered}
$$

Para el cálculo del VAN tenemos varias opciones que se presentan en la tabla III y que permiten calificar financieramente la adquisición del nuevo equipo biomédico.

Tabla 3. Recomendaciones de acuerdo al VAN

\begin{tabular}{cc}
\hline RESULTADO VAN & RECOMENDACIÓN \\
\hline VAN positivo & Se recomienda adquirir la tecnología \\
VAN $=0$ & No se perciben muchos beneficios con la \\
adquisición de la nueva tecnología \\
VAN negativo & No se recomienda adquirir la tecnología \\
\hline
\end{tabular}

El sistema facilita el cálculo del VAN y lo hace tomando como constante el numerador de (3) para calcular el VAN acumulado para $\mathrm{n}=5$ de acuerdo con lo que se muestra en la Fig. 2.

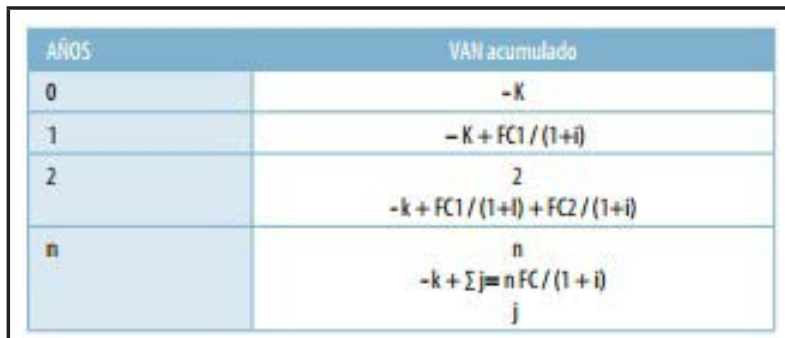

Fig. 2. VAN acumulado para $\mathrm{n}$ años. Tomado de [12].

Para realizar el análisis financiero de las diferentes propuestas de tecnología biomédica, el usuario deberá completar el formulario presentado en la Fig. 3.

Como se muestra en la Fig. 3, el usuario ingresa para cada tecnología que está evaluando (teniendo la opción de evaluar tres diferentes equipos biomédicos) el valor de la inversión inicial, el estimado de procedimientos que espera realizar con el nuevo equipo en un año, el costo del mantenimiento y metrología anual, el costo por concepto de seguro en caso de ser requerido, el costo de los consumibles que se requieren para la operación del equipo por cada procedimiento y el precio promedio del procedimiento.

El usuario debe calcular el VAN para cada año y así determinará a partir de qué año se recuperará la inversión al obtener un VAN positivo.

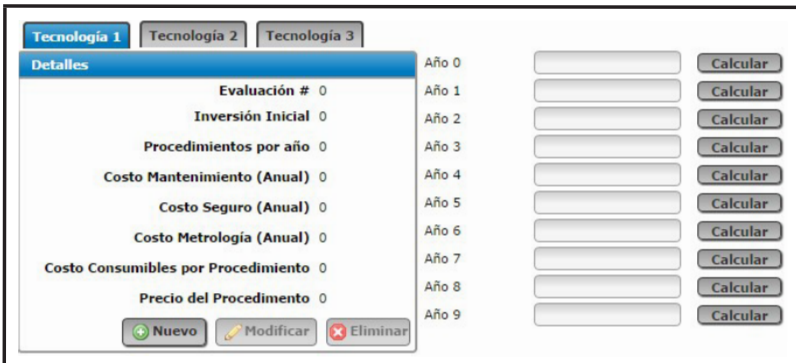

Fig. 3. Formulario para el análisis financiero para la adquisición de equipos biomédicos.

Al finalizar la evaluación económica, el usuario deberá calificar cada equipo de 1 a 5 según el tiempo de retorno de la inversión o según el menor costo de cada uno (5 calificación MUY ALTA pues el retorno es muy rápido y conveniente para la institución, 4 calificación ALTA, 3 calificación MODERADA, 2 calificación BAJA y 1 calificación DEFICIENTE).

En el caso de renovación de tecnologías o para las cuales no se puede identificar el número de procedimientos a realizar ni se establece un precio por cada uno, el sistema propone una evaluación económica por minimización de costos como se muestra en la Fig. 4.

La evaluación económica por minimización de costos presenta para cada equipo que se está considerando, el costo inicial y los costos de mantenimiento y metrología anuales para finalmente presentar una proyección a 5 años.

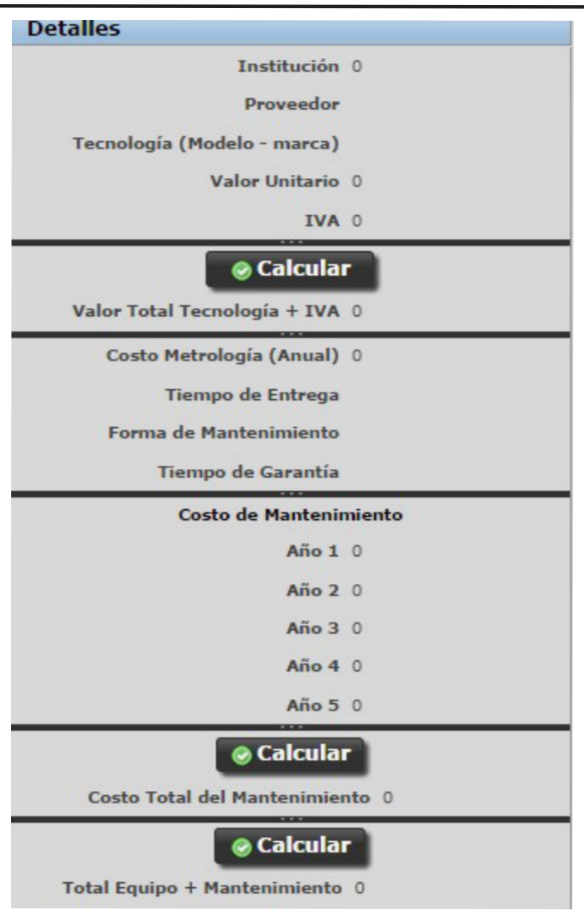

Fig. 4. Formulario para la evaluación económica por minimización de costos para la adquisición de equipos biomédicos 


\section{Evaluación clínica}

La evaluación clínica es aquella etapa en la que se acude al personal clínico para evaluar la tecnología en sus actividades y así valorar sus características, calidad, relevancia, experiencia y satisfacción [5].

Para la evaluación clínica, muchas instituciones de salud optan por realizar procesos de demostración de tecnología con el fin de familiarizar a los usuarios o potenciales futuros usuarios con el equipo biomédico.

Antes de comenzar la evaluación clínica, se deben seguir estos pasos [5]:

- Definir la cantidad de equipos que se van a evaluar.

- Definir inicio y finalización de cada evaluación (establecer un cronograma para cada equipo y proveedor).

- Definir equipo clínico potencial encargado del entrenamiento por parte del proveedor.

- Definir criterios específicos que dependiendo de la tecnología deben ser tenidos en cuenta.

Para el desarrollo de la HETES se tomaron los criterios de evaluación clínica propuestos en [5] y el usuario debe calificarlos de 1 a 5 donde 1 significa un DEFICIENTE cumplimiento del criterio para la tecnología y 5 un MUY ALTO cumplimiento del criterio para la tecnología evaluada, esto lo hará empleando el formulario presentado en la Fig. 5.

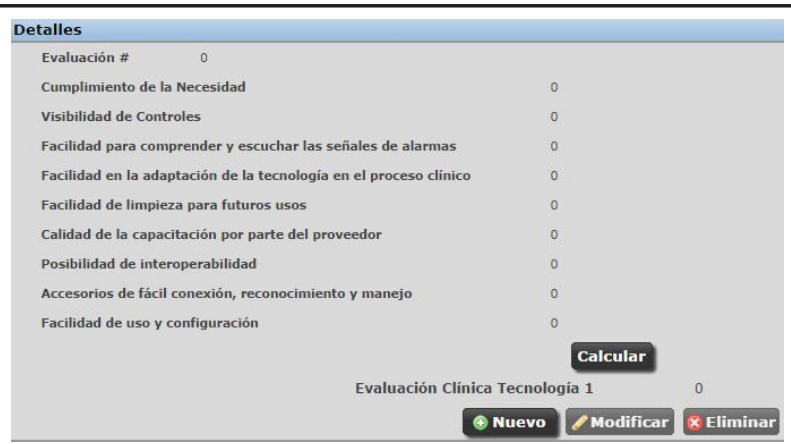

Fig. 5. Formulario para la evaluación clínica para la adquisición de equipos biomédicos

\section{Evaluación ética, social y organizacional}

La evaluación Ética, Social y Organizacional tiene como objetivos conocer de forma general cómo la incorporación y uso de una tecnología afecta a la sociedad y plantear los cuestionamientos éticos más importantes al momento de tomar la decisión de incorporar nuevas tecnologías en una organización [8].
Para la evaluación ética, social y de impacto organizacional, el usuario deberá calificar de 1 a 5 (donde 1 significa muchas repercusiones o cambios al incorporar la tecnología y 5 significa muy pocas repercusiones o cambios) cada uno de los criterios presentados en la Fig. 6.

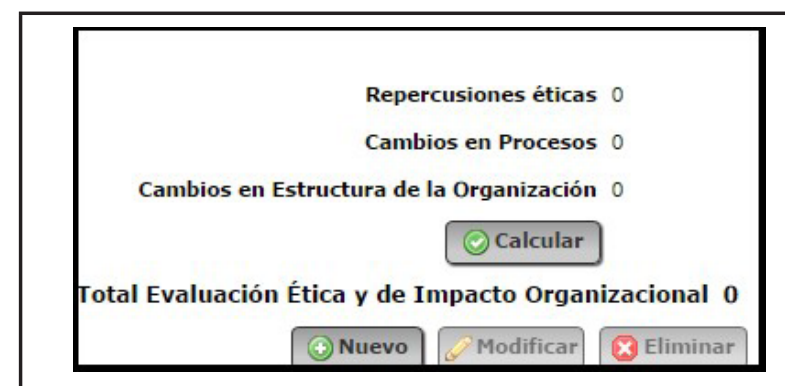

Fig. 6. Formulario de evaluación de repercusiones éticas, cambios en procesos y cambios en la estructura de la organización

\section{Recomendación}

Finalmente, el usuario podrá calcular el resultado total para las evaluaciones cuantitativas realizadas, lo que le permitirá tomar decisiones sobre la adquisición o no de una tecnología biomédica.

El sistema pondera el resultado de cada evaluación de acuerdo a la Tabla 2 y presenta un resultado general para la ETES.

Se recomienda seleccionar aquella tecnología cuyo resultado ETES es mayor. La Fig. 7 presenta el formulario que resume el proceso de evaluación de tecnologías para adquisición de equipos biomédicos.

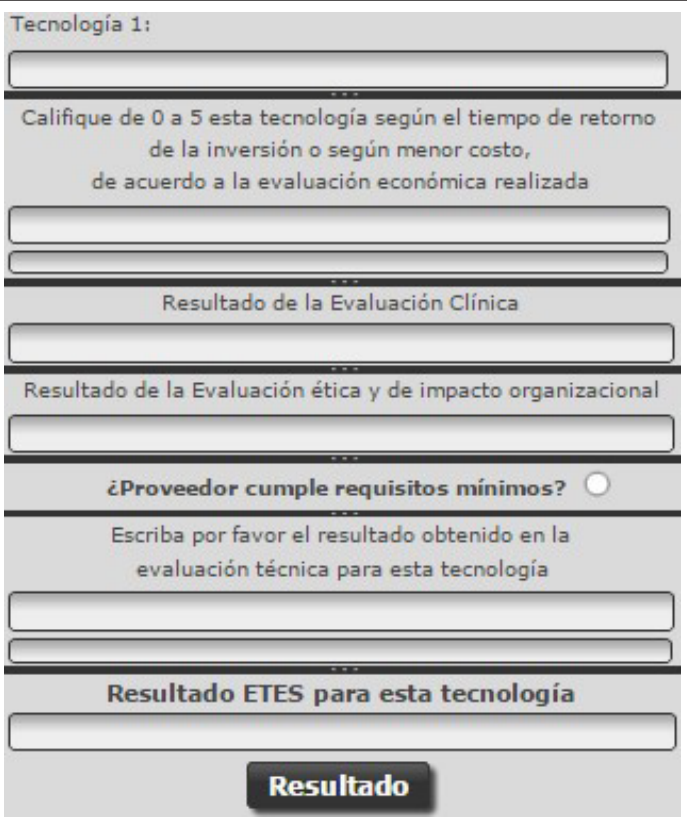

Fig. 7. Formulario de resultado de la ETES para cada equipo biomédico evaluado. 


\section{Discusión}

Al hacer un recorrido por la bibliografía de evaluación de equipos biomédicos en la etapa de adquisición, se encuentra que aunque no es un tema reciente, aún hace falta estandarizar y construir herramientas que apoyen el procedimiento.

En la identificación de actividades relevantes de ETES para equipos biomédicos en el proceso de adquisición, se encuentra que a través del tiempo la valoración económica de la tecnología en salud sigue estando vigente y es utilizado ampliamente, demostrando la necesidad de ser incluido dentro del proceso de evaluación por su importancia y relevancia en el momento de tomar decisiones de adquisición de equipos biomédicos en una IS.

Al comparar la herramienta creada con el marco de teórico para la evaluación de equipos biomédicos, se encuentra que esta aplicación es una solución integral ya que permite estandarizar el proceso, realizar una evaluación económica integral e incorpora nuevos criterios para la evaluación técnica y económica.

Criterios como la evaluación ética, social y organizacional no fueron tomados en cuenta en muchos de los procedimientos de ETES revisados, pero siempre se contó con el análisis organizacional de forma directa o indirecta, demostrando asimismo la importancia de éste.

El análisis clínico de las tecnologías sanitarias siempre estuvo presente en el procedimiento de ETES aunque en ciertas oportunidades en las instituciones no se le identifica con ese nombre concreto.

La evaluación de parámetros técnicos se encuentra a partir de mediados de la primera década del siglo XXI y se mantiene constante hasta el día el hoy, demostrando que este parámetro nació de la necesidad de un proceso de ETES más completo para así facilitar la toma de decisiones basadas en evidencia.

Al asignar los valores porcentuales de los tipos de evaluación, se encuentra que no es suficiente contar únicamente con los valores arrojados por las encuestas, sino que es necesario completar la información con procesos de evaluación de equipos biomédicos en la etapa de adquisición ya definidos y usados por IS en la ciudad de Medellín.

En la validación de la HETES se confirma la importancia de contar con procesos estandarizados de ETES en la adquisición de equipos biomédicos y se verifica la utilidad de éstas en el ámbito médico.

\section{Conclusión}

Es importante para las IS contar con un proceso estandarizado de ETES en la etapa de adquisición de equipos biomédicos y el desarrollo de esta herramienta facilita la toma de decisiones lo cual al largo plazo disminuye costos permitiendo realizar mejores inversiones en las IS.

Para futuros desarrollos, se recomienda agregar en la evaluación técnica una serie de plantillas que incluyan las especificaciones propias de cada tecnología y permitir que estas puedan ser ponderadas según su importancia para el servicio en el cual entrará en proceso el equipo biomédico adquirido.

\section{REFERENCIAS}

[1]. Rivas, R., \& Vilcahuamán, L. (2006). Ingeniería Clínica y Gestión de Tecnología en Salud: Avances y Propuestas. Lima: GRAMBS.

[2]. Escobar, N. (1999). Bvs. Recuperado el marzo de 2013, de Evaluación de Tecnologias Sanitarias: http://www.bvs.sld.cu/ revistas/san/vol3_1_99/san01199.pdf

[3]. Salud Córdoba. (23 de Agosto de 2012). Manual de Adquisición de Tecnología Biomédica. Córdoba, Colombia.

[4]. Motta Barreiro, C. M. (2009 - 2010). Sites: Editorial Salud. Recuperado el 26 de Febrero de 2012, de http://sites. paginasamarillas.com/saludeditorial/docs/Papel $\% 20 \mathrm{de} \% 201 \mathrm{a} \% 20$ Tecnologia\%20Biomedica.pdf

[5]. Carvajal, M., \& Ruiz, C. (2008). Evaluación Técnica y Clínica de Tecnología Biomédica en procesos de adquisición: un enfoque en evaluación de tecnologías en salud. Revista Ingeniería Biomédica, 34-45.

[6]. Organización Mundial de la Salud. (2012). Evaluación de las necesidades de dispositivos médicos. Organización Mundial de la Salud.

[7]. CENTEC. (2010). Evaluacíon de Tecnologías para la Salud. Documento Metodológico. México DF, Méjico: ISBN.

[8]. Gonzalez, D., \& Molina, T. (2013). Herramienta de Evaluación para la Adquisición de Equipos Biomédicos. Medellín.

[9]. Pacheco, A., Pimentel, A., Rodriguez, R., Ortiz, M., \& Salazar, R. (2002). Metodología para la Evaluación de Equipo Biomédico. Bioingeniería y Física Médica Cubana, 3(1), 2226.

[10]. Molina, T. (2012). Gestion de la Tecnologia y ETES. Medellín, Colombia.

[11]. Osteba (1999). Guia de Evaluación Económica en el sector sanitario. Departamento de sanidad. Madrid, España: Gobierno Vasco.

[12]. Villaluelga, J. L. (2008). Consideraciones antes de la compra de un equipo veterinario. Animalia, 44-47. 